\title{
THE DENNIS' SUPERTRACE AND THE HOCHSCHILD HOMOLOGY OF SUPERMATRICES
}

\author{
PAUL A. BLAGA \\ Dedicated to Professor Nicolae Teleman, on his $65^{\text {th }}$ birthday anniversary
}

\begin{abstract}
We construct, in this paper, a generalization of the Dennis trace (for matrices) to the case of the supermatrices over an arbitrary (not necessarily commutative) superalgebra with unit. By analogy with the ungraded case, we show how it is possible to use this map to construct an isomorphism from the Hochschild homology of the superalgebra to the Hochschild homology of the supermatrix algebra.
\end{abstract}

\section{The SUPERTRACE AND THE SUPERCOMMUTATOR}

We remind first a couple of things related to the $\mathbb{Z}_{2}$-grading of the supermatrix algebra $M_{p, q}(R)$. For the general material regarding the superalgebra the reader should consult the classical books of Bartocci, Bruzzo and Hernandez-Ruiperez ([3]) and Manin ([6]). A matrix $A \in M_{p, q}(R)$ is considered to be homogeneous if it can be decomposed into blocks

$$
A=\left(\begin{array}{ll}
A_{11} & A_{12} \\
A_{21} & A_{22}
\end{array}\right),
$$

where $A_{11}, A_{12}, A_{21}, A_{22}$ are matrices of type $(p, p),(p, q),(q, p),(q, q)$, respectively and all the components of one of the matrix are homogeneous of the same parity. Moreover, it is assumed that the elements of $A_{11}$ and $A_{22}$ have the same parity and the same is true for the other pair of matrices. Now, a matrix (1) satisfying this conditions is

- even if the elements of the diagonal blocks are even, while the elements from the blocks off the diagonal are odd;

- odd if the elements of the diagonal blocks are odd, while the elements from the blocks off the diagonal are even.

Now, the supertrace of matrices is the $R$-module morphism $\operatorname{str}: M_{p, q}(R) \rightarrow R$, defined on homogeneous matrices (11) by

$$
\operatorname{str}(A)=\operatorname{tr} A_{11}+(-1)^{1+|A|} \operatorname{tr} A_{22}
$$

where tr is the ordinary trace of a matrix, while with $|\cdot|$ it is denoted the parity of an element. We shall use the same notation to denote the parity of an element of the algebra $R$ and a matrix, because it will be always clear from the context what kind of object we are dealing with.

Partially supported by the Grant CEEX 130/2006 of the Romanian Ministry of Education and Research. 
It should be noted that, contrary to what one would expect, the supertrace coincides with the ordinary trace of a matrix $A$ when the matrix $A$ is odd and not even. On the other hand, for matrices from $M_{p, 0}(R)$ the supertrace is identical to the trace, no matter what parity the matrices might have. Finally, we remark that str is, indeed, an $R$-module morphism, in the sense that not only it is linear, but also preserves the parity.

Let, now, $A, B \in M_{p, q}(R)$ be two supermatrices. Their supercommutator will be

$$
\{A, B\} \doteq A \cdot B-(-1)^{|A| \cdot|B|} B \cdot A .
$$

Thus, if at least one of the two supermatrices is even, the supercommutator reduces to the ordinary commutator of two matrices. There is a difference in sign only in the case when both supermatrices are odd.

A very important property of the supercommutator, which will be useful also in the following is that it is related rather "nicely" to the supertrace.

Since the operations involved are either linear or bilinear, it is, clearly, enough to make the computations on a system of (homogeneous) generators of the algebra $M_{p, q}(R)$. A very convenient such basis is constructed from matrices of the form $E^{i j}(a)$, where $i, j \in$ $\{1, \ldots, p+q\}$, while $a$ is a homogeneous element of the algebra $A$. Here

$$
E_{k l}^{i j}(a)=\delta_{k l}^{i j} \cdot a= \begin{cases}a & \text { if } i=k, j=l, \\ 0 & \text { if } i \neq k \text { or } j \neq l .\end{cases}
$$

The parity of a matrix of the form $E^{i j}(a)$ is related to the parity of the element $a$ in a very simple manner: if $a$ is in the diagonal block components, than the two objects have the same parity, otherwise their parity is opposed. More precisely,

$$
\left|E^{i j}(a)\right|=\left\{\begin{array}{lll}
|a| & \text { if } \quad i \leq p, j \leq p & \text { or } \quad i>p, j>p \\
1+|a| & \text { if } \quad i \leq p, j>p & \text { or } \quad i>p, j \leq p .
\end{array}\right.
$$

It is not difficult to see that the family of supermatrices

$$
\left\{E^{i j}(a) \mid 1 \leq i, j \leq p+q, a \in R_{0} \text { or } a \in R_{1}\right\}
$$

is an ideal in $M_{p, q}(R)$ :

$$
E^{i j}(a) \cdot E^{k l}(b)= \begin{cases}E^{i l}(a \cdot b), & \text { if } k=l \\ 0, & \text { if } k \neq l\end{cases}
$$

Now,

$$
\begin{array}{r}
\left\{E^{i j}(a), E^{k l}(b)\right\}= \\
= \begin{cases}0, & j \neq k, i \neq l, \\
E^{i l}(a b) & j=k, i \neq l, E^{k l}(b)-(-1)^{\left|E^{i j}(a)\right| \cdot\left|E^{k l}(b)\right|} E^{k l}(b) \cdot E^{i j}(a)= \\
-(-1)^{\left|E^{i j}(a)\right|+\left|E^{k i}(b)\right|} E^{k j}(b a), & j \neq k, i=l, \\
E^{i i}(a b)-(-1)^{\left|E^{i j}(a)\right| \cdot\left|E^{j i}(b)\right|} E^{j j}(b a), & j=k, i=l .\end{cases}
\end{array}
$$


We note first that the matrices $E^{i j}(a)$ with $i \neq j$ are commutators. Let us compute now the supertrace of the supercommutator of two generating matrices, separately for each combination of indices.

We get, obviously, 0 if $j \neq k$ and $i \neq l$. If $j=k, i \neq l$, we obtain

$$
\operatorname{str}\left\{E^{i j}(a), E^{j l}(b)\right\}=\operatorname{str} E^{i l}(a b)=0 .
$$

The same is true for the case $j \neq k, i=l$. The only interesting case is the last one. Now we have

$$
\operatorname{str}\left\{E^{i j}(a), E^{j i}(b)\right\}=\operatorname{str}\left(E^{i i}(a b)-(-1)^{\left|E^{i j}(a)\right| \cdot\left|E^{j i}(b)\right|} E^{j j}(b a)\right)
$$

We have several subcases to consider here:

(i) Suppose we have $i=j$. In this case, we have $\left|E^{i i}(a)\right|=|a|,\left|E^{i i}(b)\right|=|b|$, thus,

$$
\begin{aligned}
\left\{E^{i i}(a), E^{i i}(b)\right\} & =E^{i i}(a b)-(-1)^{|a| \cdot|b|} E^{i i}(b a)=E^{i i}\left(a b-(-1)^{|a| \cdot|b|} b a\right) \\
& =E^{i i}(\{a, b\}),
\end{aligned}
$$

therefore

$$
\operatorname{str}\left\{E^{i i}(a), E^{i i}(b)\right\}=\operatorname{str} E^{i i}(\{a, b\})= \begin{cases}\{a, b\}, & i \leq p \\ (-1)^{1+|a|+|b|}\{a, b\}, & i>p .\end{cases}
$$

(ii) $i<j \leq p$. In this case we have $\left|E^{i j}(a)\right|=|a|,\left|E^{j i}(b)\right|=|b|$, therefore

$$
\left\{E^{i j}(a), E^{j i}(b)\right\}=E^{i i}(a b)-(-1)^{|a| \cdot|b|} E^{j j}(b a),
$$

hence

$$
\begin{aligned}
\operatorname{str}\left\{E^{i j}(a), E^{j i}(b)\right\} & =\operatorname{str} E^{i i}(a b)-(-1)^{|a| \cdot|b|} \operatorname{str} E^{j j}(b a)= \\
& =a b-(-1)^{|a| \cdot|b|} b a=\{a, b\} .
\end{aligned}
$$

(iii) $i \leq p<j$. Now $\left|E^{i j}(a)\right|=1+|a|,\left|E^{j i}(b)\right|=1+|b|$, and then

$$
\left\{E^{i j}(a), E^{j i}(b)\right\}=E^{i i}(a b)-(-1)^{1+|a|+|b|+|a| \cdot|b|} E^{j j}(b a)
$$

and

$$
\begin{aligned}
\operatorname{str}\left\{E^{i j}(a), E^{j i}(b)\right\} & =\operatorname{str} E^{i i}(a b)-(-1)^{1+|a|+|b|+|a| \cdot|b|} \operatorname{str} E^{j j}(b a)= \\
& =a b-(-1)^{1+|a|+|b|+|a| \cdot|b|} \cdot(-1)^{1+|a|+|b|} b a= \\
& =a b-(-1)^{|a| \cdot|b|} b a=\{a, b\} .
\end{aligned}
$$

(iv) $p<i<j$. In this situation, $\left|E^{i j}(a)\right|=|a|,\left|E^{j i}(b)\right|=|b|$, therefore

$$
\left\{E^{i j}(a), E^{j i}(b)\right\}=E^{i i}(a b)-(-1)^{|a| \cdot|b|} E^{j j}(b a),
$$

but

$$
\begin{aligned}
\operatorname{str}\left\{E^{i j}(a), E^{j i}(b)\right\} & =\operatorname{str} E^{i i}(a b)-(-1)^{|a| \cdot|b|} \operatorname{str} E^{j j}(b a)= \\
& =(-1)^{1+|a|+|b|}\left(a b-(-1)^{|a| \cdot|b|} b a\right)=(-1)^{1+|a|+|b|}\{a, b\} .
\end{aligned}
$$


(v) $j<i \leq p$. This case is identical to the case (ii).

(vi) $j \leq p<i$. We have now, as in the case (iii), $\left|E^{i j}(a)\right|=1+|a|,\left|E^{j i}(b)\right|=1+|b|$, therefore

$$
\left\{E^{i j}(a), E^{j i}(b)\right\}=E^{i i}(a b)-(-1)^{1+|a|+|b|+|a| \cdot|b|} E^{j j}(b a),
$$

but

$$
\begin{aligned}
\operatorname{str}\left\{E^{i j}(a), E^{j i}(b)\right\} & =\operatorname{str} E^{i i}(a b)-(-1)^{1+|a|+|b|+|a| \cdot|b|} \operatorname{str} E^{j j}(b a)= \\
& =(-1)^{1+|a|+|b|} a b-(-1)^{1+|a|+|b|+|a| \cdot|b|} b a= \\
& =(-1)^{1+|a|+|b|}\{a, b\} .
\end{aligned}
$$

(vii) $p<j<i$. This case is identical to the case (iv).

\section{The Hochschild homology of Superalgebras}

The Hochschild complex for superalgebras (Kassel, 1986), is very similar to the analogous complex for ungraded case. Namely, the chain groups are, as in the classical case, $C_{m}(R)=$ $R^{\otimes m+1}$, where, of course, the tensor product should be understood in the graded sense, while the face maps and degeneracies are given by

$$
\begin{gathered}
\delta_{i}^{m}\left(a_{0} \otimes \cdots \otimes a_{m}\right)=a_{0} \otimes \cdots \otimes a_{i} a_{i+1} \otimes \ldots a_{n}, \quad \text { if } \quad 0 \leq i<m, \\
\delta_{m}^{m}\left(a_{0} \otimes \cdots \otimes a_{m}\right)=(-1)^{\left|a_{m}\right|\left(\left|a_{0}\right|+\cdots+\mid a_{m-1}\right)} a_{m} a+0 \otimes a_{1} \otimes \cdots \otimes a_{m-1}, \\
s_{i}^{m}\left(a_{0} \otimes \cdots \otimes a_{m}\right)=a_{0} \otimes \cdots \otimes a_{i} \otimes 1 \otimes a_{i+1} \otimes \cdots \otimes a_{m}, \quad 0 \leq i \leq m .
\end{gathered}
$$

Now the differential is defined in the usual way, meaning $d^{m}: C_{m}(R) \rightarrow C_{m-1}(R)$,

$$
d^{m}=\sum_{i=0}^{m}(-1)^{i} \delta_{i}^{m} .
$$

and the Hochschild homology of the superalgebra is just the homology of the complex $(C(R), d)$. In particular, it is easy to see that for any superalgebra $R$ we have

$$
H_{0}(R)=R /\{R, R\}
$$

where $\{R, R\}$ is the subspace generated by the supercommutators.

\section{The Dennis supertrace And its properties}

If $A^{1}$ and $A^{2}$ are two square matrices over an arbitrary algebra $R$, then their product is

$$
\left(A^{1} \cdot A^{2}\right)_{i j}=\sum_{k=1}^{n} A_{i k}^{1} \cdot A_{k j}^{2},
$$

therefore, the trace of the product is

$$
\operatorname{tr}\left(A^{1} \cdot A^{2}\right)=\sum_{i=1}^{n} \sum_{k=1}^{n} A_{i k}^{1} \cdot A_{k i}^{2} .
$$


Completely analogously, the trace of the product of $m+1 \geq 2$ matrices is

$$
\operatorname{tr}\left(A^{0} \cdot A^{1} \ldots A^{m}\right)=\sum_{i_{0}=1}^{n} \sum A_{i_{0} i_{1}}^{0} \cdot A_{i_{1} i_{2}}^{1} \ldots A_{i_{m-1} i_{m}}^{m-1} A_{i_{m} i_{0}}^{m},
$$

where the second sum is taken after all the possible values of the indices $i_{1}, \ldots, i_{m} \in$ $\{1, \ldots, n\}$.

Now, the very natural idea of Dennis was to define a generalized trace map (which is now often called Dennis trace),

$$
\operatorname{Tr}^{m}: M_{n}(R)^{\otimes m+1} \rightarrow R^{\otimes m+1}
$$

putting

$$
\operatorname{Tr}^{m}\left(A^{0} \otimes A^{1} \otimes \cdots \otimes A^{m}\right)=\sum_{i_{0}=1}^{n} \sum A_{i_{0} i_{1}}^{0} \otimes A_{i_{1} i_{2}}^{1} \otimes \cdots \otimes A_{i_{m-1} i_{m}}^{m-1} A_{i_{m} i_{0}}^{m},
$$

with the second summation sign having the same significance as above. Dennis used the generalized trace to construct an isomorphism between the Hochschild homology of the matrix algebra over an algebra $R$ and that of the algebra itself.

The Dennis' construction can be carried out also in the case of superalgebras if we replace the trace with the supertrace and we pay attention to the signs.

Namely, it is easy to see that the supertrace of a product of two homogeneous supermatrices of type $(p, q)$ over a superalgebra $R$

$$
\operatorname{str}\left(A^{1} \cdot A^{2}\right)=\sum_{i=1}^{p} \sum_{k=1}^{p+q} A_{i k}^{1} \cdot A_{k i}^{2}+(-1)^{1+\left|A^{1}\right|+\left|A^{2}\right|} \sum_{i=p+1}^{p+q} \sum_{k=1}^{p+q} A_{i k}^{1} \cdot A_{k i}^{2},
$$

while for $m+1$ supermatrices we have

$$
\begin{aligned}
\operatorname{str}\left(A^{0} \cdot A^{1} \ldots A^{m}\right) & =\sum_{i_{0}=1}^{p} \sum A_{i_{0} i_{1}}^{0} \cdot A_{i_{1} i_{2}}^{1} \ldots A_{i_{m-1} i_{m}}^{m-1} A_{i_{m} i_{0}}^{m}+ \\
& +(-1)^{1+\left|A^{0}\right|+\cdots+\left|A^{m}\right|} \sum_{i_{0}=p+1}^{p+q} \sum A_{i_{0} i_{1}}^{0} \cdot A_{i_{1} i_{2}}^{1} \ldots A_{i_{m-1} i_{m}}^{m-1} A_{i_{m} i_{0}}^{m},
\end{aligned}
$$

where, as above, the second sum in each term is taken after all the values of the indices $i_{1}, \ldots, i_{m} \in\{1, \ldots, p+q\}$.

Now, it is clear that to have a consistent generalization of the Dennis trace for the $\mathbb{Z}_{2}$-graded case we should put (for homogeneous supermatrices)

$$
\begin{aligned}
& \operatorname{Str}^{m}\left(A^{0} \otimes A^{1} \otimes \cdots \otimes A^{m}\right)=\sum_{i_{0}=1}^{p} \sum A_{i_{0} i_{1}}^{0} \otimes A_{i_{1} i_{2}}^{1} \otimes \cdots \otimes A_{i_{m}-1 i_{m}}^{m-1} A_{i_{m} i_{0}}^{m}+ \\
& +(-1)^{1+\left|A^{0}\right|+\cdots+\left|A^{m}\right|} \sum_{i_{0}=p+1}^{p+q} \sum A_{i_{0} i_{1}}^{0} \otimes A_{i_{1} i_{2}}^{1} \otimes \cdots \otimes A_{i_{m-1} i_{m}}^{m-1} A_{i_{m} i_{0}}^{m} .
\end{aligned}
$$


We shall call this generalized supertrace the Dennis supertrace map.

It is convenient to work, as before, with the (homogeneous) generators $E^{i j}(a)$ of the supermatrix algebra $M_{p, q}(R)$. The nice thing about them is that the Dennis supertace can be written down very easily, for these generators, because, as one can see immediately,

$$
\operatorname{Str}^{m}\left(E^{i_{0} j_{0}}\left(a_{0}\right) \otimes \cdots \otimes E^{i_{m} j_{m}}\left(a_{m}\right)\right) \neq 0
$$

if and only if we have

$$
j_{0}=i_{1}, j_{1}=i_{2}, \ldots, j_{m-1}=i_{m}, j_{m}=i_{0},
$$

therefore we shall suppose all the time that these conditions are fulfilled. Now, it is easy to see that

Proposition 1. The Dennis supertrace can be written on the homogeneous generators as

$$
\operatorname{Str}^{m}\left(E^{i_{0} i_{1}}\left(a_{0}\right) \otimes \cdots \otimes E^{i_{m} i_{0}}\left(a_{m}\right)\right)= \begin{cases}a_{0} \otimes \cdots \otimes a_{m}, & i_{0} \leq p, \\ (-1)^{1+\left|a_{0}\right|+\cdots+\left|a_{m}\right|} a_{0} \otimes \cdots \otimes a_{m}, & i_{0}>p .\end{cases}
$$

Proof. Clearly, the only thing that calls for a justification is the fact that

$$
\left|E^{i_{0} i_{1}}\left(a_{0}\right)\right|+\cdots+\left|E^{i_{m} i_{0}}\left(a_{m}\right)\right|=\left|a_{0}\right|+\cdots+\left|a_{m}\right| .
$$

But this follows immediately if we notice that

$$
E^{i_{0} i_{1}}\left(a_{0}\right) \cdot E^{i_{1} i_{2}}\left(a_{1}\right) \ldots E^{i_{m} i_{0}}\left(a_{m}\right)=E^{i_{0} i_{0}}\left(a_{0} \cdot a_{1} \ldots a_{m}\right),
$$

therefore, on the one hand

$$
\left|E^{i_{0} i_{1}}\left(a_{0}\right) \cdot E^{i_{1} i_{2}}\left(a_{1}\right) \ldots E^{i_{m} i_{0}}\left(a_{m}\right)\right|=\left|E^{i_{0} i_{1}}\left(a_{0}\right)\right|+\cdots+\left|E^{i_{m} i_{0}}\left(a_{m}\right)\right|
$$

and, on the other hand,

$\left|E^{i_{0} i_{1}}\left(a_{0}\right) \cdot E^{i_{1} i_{2}}\left(a_{1}\right) \ldots E^{i_{m} i_{0}}\left(a_{m}\right)\right|=\left|E^{i_{0} i_{0}}\left(a_{0} \cdot a_{1} \ldots a_{m}\right)\right|=\left|a_{0} \cdot a_{1} \ldots a_{m}\right|=\left|a_{0}\right|+\cdots+\left|a_{m}\right|$,

where, when we wrote the second equality, we took into account the fact that the only non-vanishing element of the matrix $E^{i_{0} i_{0}}\left(a_{0} \cdot a_{1} \ldots a_{m}\right)$ is on the diagonal, therefore the parity of the matrix is equal to the parity of that element.

Theorem 1. The family of mappings $\left\{\operatorname{Str}^{m}: M_{p, q}(R)^{\otimes m+1} \rightarrow R^{\otimes m+1}\right\}$ defines a chain morphism between the Hochschild complex of the algebra $M_{p, q}(R)$ and the Hochschild complex of the ground algebra $R$.

Proof. Clearly, each Dennis supertrace is a linear map. All we have to do is to show that the Dennis traces commute with the face maps of the two Hochschild complexes, i.e. with the operators $\delta_{k}^{m}, k=0, \ldots, m$. Again, it is enough to verify for elements of the form

$$
E^{i_{0} i_{1}} \otimes \cdots \otimes E^{i_{m} i_{0}} \text {. }
$$

We shall discuss first the case $k<m$. As we saw above, the Dennis supetrace, calculated on such an element is

$$
\operatorname{Str}^{m}\left(E^{i_{0} i_{1}}\left(a_{0}\right) \otimes \cdots \otimes E^{i_{m} i_{0}}\left(a_{m}\right)\right)= \begin{cases}a_{0} \otimes \cdots \otimes a_{m}, & i_{0} \leq p \\ (-1)^{1+\left|a_{0}\right|+\cdots+\left|a_{m}\right|} a_{0} \otimes \cdots \otimes a_{m}, & i_{0}>p\end{cases}
$$


Thus, we have

$$
\begin{aligned}
\delta_{k}^{m} \operatorname{Str}^{m}\left(E^{i_{0} i_{1}}\left(a_{0}\right) \otimes \cdots \otimes E^{i_{m} i_{0}}\left(a_{m}\right)\right)= \\
\quad= \begin{cases}a_{0} \otimes \ldots a_{k} a_{k+1} \otimes \cdots \otimes a_{m}, & i_{0} \leq p, \\
(-1)^{1+\left|a_{0}\right|+\cdots+\left|a_{m}\right|} a_{0} \otimes \ldots a_{k} a_{k+1} \otimes \cdots \otimes a_{m}, & i_{0}>p .\end{cases}
\end{aligned}
$$

On the other hand,

$$
\begin{aligned}
& \delta_{k}^{m}\left(E^{i_{0} i_{1}}\left(a_{0}\right) \otimes \cdots \otimes E^{i_{m} i_{0}}\left(a_{m}\right)\right)= \\
& E^{i_{0} i_{1}}\left(a_{0}\right) \otimes \ldots E^{i_{k} i_{k+1}}\left(a_{k}\right) E^{i_{k+1} i_{k+2}}\left(a_{k+1}\right) \otimes \cdots \otimes E^{i_{m} i_{0}}\left(a_{m}\right)= \\
& E^{i_{0} i_{1}}\left(a_{0}\right) \otimes \ldots E^{i_{k} i_{k+2}}\left(a_{k} a_{k+1}\right) \otimes \cdots \otimes E^{i_{m} i_{0}}\left(a_{m}\right),
\end{aligned}
$$

therefore

$$
\begin{aligned}
\operatorname{Str}^{m-1} \delta_{k}^{m}( & \left.E^{i_{0} i_{1}}\left(a_{0}\right) \otimes \cdots \otimes E^{i_{m} i_{0}}\left(a_{m}\right)\right)= \\
= & \begin{cases}a_{0} \otimes \ldots a_{k} a_{k+1} \otimes \cdots \otimes a_{m}, & i_{0} \leq p, \\
(-1)^{1+\left|a_{0}\right|+\cdots+\left|a_{m}\right|} a_{0} \otimes \ldots a_{k} a_{k+1} \otimes \cdots \otimes a_{m}, & i_{0}>p . \\
\quad=\delta_{k}^{m} \operatorname{Str}^{m}\left(E^{i_{0} i_{1}}\left(a_{0}\right) \otimes \cdots \otimes E^{i_{m} i_{0}}\left(a_{m}\right)\right),\end{cases}
\end{aligned}
$$

where we have use the fact that $\left|a_{k} a_{k+1}\right|=\left|a_{k}\right|+\left|a_{k+1}\right|$. The only case that needs extra work is the case $k=m$. In this case we have, on the one hand,

$$
\begin{aligned}
& \delta_{m}^{m} \operatorname{Str}^{m}\left(E^{i_{0} i_{1}}\left(a_{0}\right) \otimes \cdots \otimes E^{i_{m} i_{0}}\left(a_{m}\right)\right)= \\
& \begin{cases}(-1)^{\left|a_{m}\right| \sum_{i=0}^{m-1}\left|a_{i}\right|} a_{0} \otimes \ldots a_{k} a_{k+1} \otimes \cdots \otimes a_{m}, & i_{0} \leq p, \\
(-1)^{1+\sum_{i=0}^{m}\left|a_{i}\right|+\left|a_{m}\right| \sum_{i=0}^{m-1}\left|a_{i}\right|} a_{0} \otimes \ldots a_{k} a_{k+1} \otimes \cdots \otimes a_{m}, & i_{0}>p .\end{cases}
\end{aligned}
$$

On the other hand,

$$
\begin{aligned}
& \operatorname{Str}^{m-1} \delta_{m}^{m}\left(E^{i_{0} i_{1}}\left(a_{0}\right) \otimes \cdots \otimes E^{i_{m} i_{0}}\left(a_{m}\right)\right)=
\end{aligned}
$$

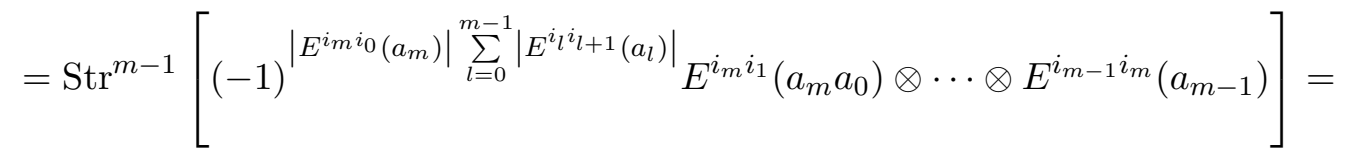

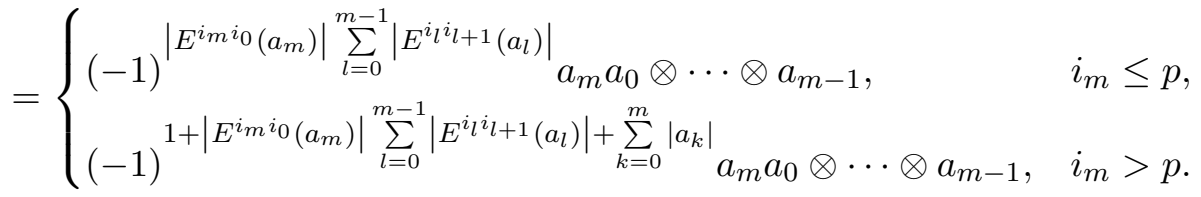

Now, to prove that $\operatorname{Str}^{m-1} \delta_{m}^{m}=\delta_{m}^{m} \operatorname{Str}^{m}$, we have to consider several cases. 
(i) $i_{0} \leq p, i_{m} \leq p$. In this case we have to prove that

$$
\left|a_{m}\right| \sum_{k=0}^{m-1}\left|a_{k}\right|=\left|E^{i_{m} i_{0}}\left(a_{m}\right)\right| \sum_{l=0}^{m-1}\left|E^{i_{l} i_{l+1}}\left(a_{l}\right)\right| .
$$

But, since we have $i_{0}, i_{m} \leq p$, it follows that $\left|E^{i_{m} i_{0}}\left(a_{m}\right)\right|=\left|a_{m}\right|$ and we have already seen that

$$
\sum_{k=0}^{m-1}\left|a_{k}\right|=\sum_{l=0}^{m-1}\left|E^{i_{l} i_{l+1}}\left(a_{l}\right)\right|
$$

(ii) $i_{0} \leq p, i_{m}>p$. Now the identity we have to prove is

$$
\left|a_{m}\right| \sum_{k=0}^{m-1}\left|a_{k}\right|=\underbrace{1+\sum_{k=0}^{m}\left|a_{k}\right|+\left|E^{i_{m} i_{0}}\left(a_{m}\right)\right| \sum_{l=0}^{m-1}\left|E^{i_{l} i_{l+1}}\left(a_{l}\right)\right|}_{R H S} .
$$

In this case, $\left|E^{i_{m} i_{0}}\left(a_{m}\right)\right|=1+\left|a_{m}\right|$. Moreover, we have

$$
\sum_{l=0}^{m-1}\left|E^{i_{l} i_{l+1}}\left(a_{l}\right)\right|=\sum_{l=0}^{m}\left|E^{i_{l} i_{l+1}}\left(a_{l}\right)\right|-\left|E^{i_{m} i_{0}}\left(a_{m}\right)\right|=\sum_{k=0}^{m}\left|a_{k}\right|-1-\left|a_{m}\right|=1+\sum_{k=0}^{m-1}\left|a_{k}\right| .
$$

It follows, therefore, that

$$
\begin{aligned}
R H S=1+\sum_{k=0}^{m}\left|a_{k}\right|+ & \left(1+\left|a_{m}\right|\right)\left(1+\sum_{k=0}^{m-1}\left|a_{k}\right|\right)= \\
1 & +\sum_{k=0}^{m}\left|a_{k}\right|+1+\sum_{k=0}^{m-1}\left|a_{k}\right|+\left|a_{m}\right|+\left|a_{m}\right| \sum_{k=0}^{m-1}\left|a_{k}\right|=\left|a_{m}\right| \sum_{k=0}^{m-1}\left|a_{k}\right|,
\end{aligned}
$$

so the identity is proven.

(iii) $i_{0}>p, i_{m} \leq p$. We have to show that

$$
1+\sum_{k=0}^{m}\left|a_{k}\right|+\left|a_{m}\right| \sum_{k=0}^{m-1}\left|a_{k}\right|=\underbrace{\left|E^{i_{m} i_{0}}\left(a_{m}\right)\right| \sum_{l=0}^{m-1}\left|E^{i_{l} i_{l+1}}\left(a_{l}\right)\right|}_{\text {RHS }} .
$$

The same reasoning we did before ensure us that we have $\left|E^{i_{m} i_{0}}\left(a_{m}\right)\right|=1+\left|a_{m}\right|$ and

$$
\sum_{l=0}^{m-1}\left|E^{i_{l} i_{l+1}}\left(a_{l}\right)\right|=1+\sum_{k=0}^{m-1}\left|a_{k}\right|
$$


hence

$$
\begin{aligned}
R H S & =\left(1+\left|a_{m}\right|\right)\left(1+\sum_{k=0}^{m-1}\left|a_{k}\right|\right)=1+\sum_{k=0}^{m-1}\left|a_{k}\right|+\left|a_{m}\right| \sum_{k=0}^{m-1}\left|a_{k}\right|+\left|a_{m}\right|= \\
& =1+\sum_{k=0}^{m}\left|a_{k}\right|+\left|a_{m}\right| \sum_{k=0}^{m-1}\left|a_{k}\right|,
\end{aligned}
$$

so we are done.

(iv) $i_{0}>p, i_{m}>p$. The required identity reads

$$
1+\sum_{k=0}^{m}\left|a_{k}\right|+\left|a_{m}\right| \sum_{k=0}^{m-1}\left|a_{k}\right|=1+\sum_{k=0}^{m-1}\left|a_{k}\right|+\left|E^{i_{m} i_{0}}\left(a_{m}\right)\right| \sum_{l=0}^{m-1}\left|E^{i_{l} i_{l+1}}\left(a_{l}\right)\right|
$$

or, which is the same,

$$
\left|a_{m}\right| \sum_{k=0}^{m-1}\left|a_{k}\right|=\left|E^{i_{m} i_{0}}\left(a_{m}\right)\right| \sum_{l=0}^{m-1}\left|E^{i_{l} i_{l+1}}\left(a_{l}\right)\right|,
$$

which is obvious, since in this case $\left|E^{i_{m} i_{0}}\left(a_{m}\right)\right|=\left|a_{m}\right|$.

It is pretty clear that the Dennis trace maps are onto. In fact, we can consider the map

$$
\text { inc }: R \rightarrow M_{p, q}(R)
$$

given by $\operatorname{inc}(a)=E^{11}(a)$. This map, which is, obviously, a linear morphism $\left(\left|E^{11}(a)\right|=\right.$ $|a|)$, can be extended, for each natural $m$, to a morphism

$$
\begin{gathered}
\operatorname{Inc}^{m}: R^{\otimes m+1} \rightarrow M_{p, q}(R)^{\otimes m+1}, \\
\operatorname{Inc}^{m}\left(a_{0} \otimes \cdots \otimes a_{m}\right)=E^{11}\left(a_{0}\right) \otimes \cdots \otimes E^{11}\left(a_{m}\right) .
\end{gathered}
$$

It can be shown immediately that

Proposition 2. The family of maps $\left\{\operatorname{Inc}^{m}: R^{\otimes m+1} \rightarrow M_{p, q}(R)^{\otimes m+1}, m \in \mathbb{N}\right\}$, is a chain map from the Hochschild complex of $R$ to the Hochschild complex of $M_{p, q}(R)$, which is a splitting of the Dennis supertrace.

\section{The Hochschild homology of $M_{p, q}(R)$}

Inc is a right inverse of the Dennis supertrace, but, obviously, it is not, also, a right inverse, so the Hochschild complexes of $M_{p, q}(R)$ and $R$ are not isomorphical. We shall prove that, however, the supertrace induces an isomorphism in homology. To prove this, it is enough to verify that Inc is a left quasi-inverse of the supetrace, i.e.

Theorem 2. There is a chain homotopy $h: C\left(M_{p, q}(R)\right) \rightarrow C\left(M_{p, q}(R)\right)$ such that

$$
d \circ h+h \circ d=\operatorname{Id}-\operatorname{Inc} \circ \operatorname{Str} .
$$


Proof. We shall define the homotopy exactly as in the classical (non-graded) case and we shall check that it does the job equally in the supercase. Thus, let us consider

$$
h=\sum_{l=0}^{m}(-1)^{l} h_{l}: M_{p, q}(R)^{\otimes m+1} \rightarrow M_{p, q}(R)^{\otimes m+2},
$$

with

$$
\begin{aligned}
& h_{l}\left(E^{i_{0} i_{1}}\left(a_{0}\right) \otimes \cdots \otimes E^{i_{m} i_{0}}\left(a_{m}\right)\right)= \\
& \quad=E^{i_{0} 1}\left(a_{0}\right) \otimes E^{11}\left(a_{1}\right) \otimes \cdots \otimes E^{11}\left(a_{l}\right) \otimes E^{1 i_{l+1}}(1) \otimes E^{i_{l+1} i_{l+2}}\left(a_{l+1}\right) \otimes \cdots \otimes E^{i_{m} i_{0}}\left(a_{m}\right)
\end{aligned}
$$

Let us, verify, first, that it works for the particular case of $m=0$. We have

$$
\begin{gathered}
h_{0}\left(E^{i_{0} j_{0}}\left(a_{0}\right)\right)=E^{i_{0} 1}\left(a_{0}\right) \otimes E^{1 j_{0}}(1) . \\
d^{1} \circ h_{0}\left(E^{i_{0} j_{0}}\left(a_{0}\right)\right)=\delta_{0}^{1} \circ h_{0}\left(E^{i_{0} j_{0}}\left(a_{0}\right)\right)-\delta_{1}^{1} \circ h_{0}\left(E^{i_{0} j_{0}}\left(a_{0}\right)\right)= \\
=\delta_{0}^{1}\left(E^{i_{0} 1}\left(a_{0}\right) \otimes E^{1 j_{0}}(1)\right)-\delta_{1}^{1}\left(E^{i_{0} 1}\left(a_{0}\right) \otimes E^{1 j_{0}}(1)\right)= \\
=\left\{\begin{array}{ll}
E^{i_{0} j_{0}}\left(a_{0}\right) & i_{0} \neq j_{0} \\
E^{i_{0} i_{0}}\left(a_{0}\right)-(-1)^{\left|E^{i_{0}}\left(a_{0}\right)\right| \cdot\left|E^{1 i_{0}}(1)\right|} E^{11}\left(a_{0}\right) & i_{0}=j_{0}
\end{array}=\right. \\
= \begin{cases}E_{0}^{i_{0} j_{0}}\left(a_{0}\right) & i_{0}=j_{0} \leq p,=(\operatorname{Id}-\operatorname{Inc} \circ \operatorname{Str})\left(E^{i_{0} j_{0}}\left(a_{0}\right)\right) . \\
E^{i_{0} i_{0}}\left(a_{0}\right)-E^{11}\left(a_{0}\right) & i_{0}=j_{0}>p \\
E^{i_{0} i_{0}}\left(a_{0}\right)-(-1)^{1+\left|a_{0}\right|} E^{11}\left(a_{0}\right)\end{cases}
\end{gathered}
$$

Thus, the claim is true at the lowest level. Take now an arbitrary $m \in \mathbb{N}$. Let us compute first $\delta_{m+1}^{m+1} \circ h_{m}$. We have

$$
\begin{aligned}
& \delta_{m+1}^{m+1} \circ h_{m}\left(E^{i_{0} i_{1}}\left(a_{0}\right) \otimes \cdots \otimes E^{i_{m} i_{0}}\left(a_{m}\right)\right)= \\
& \delta_{m+1}^{m+1}\left(E^{i_{0} 1}\left(a_{0}\right) \otimes E^{11}\left(a_{1}\right) \otimes \cdots \otimes E^{11}\left(a_{m}\right) \otimes E^{1 i_{0}}(1)\right)= \\
& =(-1)^{\left|E^{1 i_{0}}(1)\right|\left(\left|E^{i_{0} 1}\left(a_{0}\right)\right|+\sum_{k=1}^{m}\left|a_{k}\right|\right)} E^{11}\left(a_{0}\right) \otimes E^{11}\left(a_{1}\right) \otimes \cdots \otimes E^{11}\left(a_{m}\right)= \\
& = \begin{cases}E^{11}\left(a_{0}\right) \otimes E^{11}\left(a_{1}\right) \otimes \cdots \otimes E^{11}\left(a_{m}\right), & i_{0} \leq p, \\
(-1)^{1+\sum_{k=0}^{m}\left|a_{k}\right|} E^{11}\left(a_{0}\right) \otimes E^{11}\left(a_{1}\right) \otimes \cdots \otimes E^{11}\left(a_{m}\right), & i_{0}>p .\end{cases}
\end{aligned}
$$

On the other hand,

$$
\operatorname{Str}^{m}\left(E^{i_{0} i_{1}}\left(a_{0}\right) \otimes \cdots \otimes E^{i_{m} i_{0}}\left(a_{m}\right)\right)= \begin{cases}a_{0} \otimes \cdots \otimes a_{m}, & i_{0} \leq p, \\ (-1)^{1+\sum_{k=0}^{m}\left|a_{k}\right|} a_{0} \otimes \cdots \otimes a_{m}, & i_{0}>p .\end{cases}
$$

It follows then, immediately, that

$$
\delta_{m+1}^{m+1} \circ h_{m}=\operatorname{Inc}^{m} \circ \operatorname{Str}^{m} .
$$


Moreover, we have

$$
\begin{array}{r}
\delta_{0}^{m+1} \circ h_{0}\left(E^{i_{0} i_{1}}\left(a_{0}\right) \otimes \cdots \otimes E^{i_{m} i_{0}}\left(a_{m}\right)\right)= \\
=\delta_{0}^{m+1}\left(E^{i_{0} 1}\left(a_{0}\right) \otimes E^{1 i_{1}}(1) \otimes E^{i_{1} i_{2}}\left(a_{1}\right) \otimes \cdots \otimes E^{i_{m} i_{0}}\left(a_{m}\right)\right)= \\
E^{i_{0} i_{1}}\left(a_{0}\right) \otimes \cdots \otimes E^{i_{m} i_{0}}\left(a_{m}\right)= \\
=\operatorname{Id}\left(E^{i_{0} i_{1}}\left(a_{0}\right) \otimes \cdots \otimes E^{i_{m} i_{0}}\left(a_{m}\right)\right) .
\end{array}
$$

Now, exactly as in the classical case, one verifies immediately that if $1 \leq l \leq m$ then

$$
\delta_{l}^{m+1} \circ h_{l}=\delta_{l}^{m+1} \circ h_{l-1},
$$

while, if $k<l \leq m$, then

$$
\delta_{k}^{m+1} \circ h_{l}=h_{l-1} \circ \delta_{k}^{m}
$$

and, also, if $k \geq l$, then

$$
\delta_{k}^{m+1} \circ h_{l}=h_{l} \circ \delta_{k-1}^{m}
$$

To summarize, we have the following set of relations:

$$
\begin{aligned}
\delta_{m+1}^{m+1} \circ h_{m} & =\mathrm{Inc}^{m} \circ \mathrm{Str}^{m} ; \\
\delta_{0}^{m+1} \circ h_{0} & =\mathrm{Id} ; \\
\delta_{l}^{m+1} \circ h_{l} & =\delta_{l}^{m+1} \circ h_{l-1}, \quad \text { if } 1 \leq l \leq m ; \\
\delta_{k}^{m+1} \circ h_{l} & =h_{l-1} \circ \delta_{k}^{m}, \quad \text { if } k<l \leq m ; \\
\delta_{k}^{m+1} \circ h_{l} & =h_{l} \circ \delta_{k-1}^{m}, \quad \text { if } k \geq l+2 . .
\end{aligned}
$$


We have everything we need to prove our assertion:

$$
\begin{aligned}
& d^{m+1} \circ h^{m}+h^{m} \circ d^{m}=\sum_{k=0}^{m+1} \sum_{l=0}^{m}(-1)^{k+l} \delta_{k}^{m+1} \circ h_{l}^{m}+\sum_{k=0}^{m} \sum_{l=0}^{m-1}(-1)^{k+l} h_{l}^{m} \circ \delta_{k}^{m}= \\
& =\delta_{0}^{m+1} \circ h_{0}-\delta_{m+1}^{m+1} \circ h_{m}+\sum_{l=1}^{m} \delta_{l}^{m+1} \circ h_{l}-\sum_{l=1}^{m} \delta_{l}^{m+1} \circ h_{l-1}+ \\
& +\sum_{k=0}^{l-1} \sum_{l=1}^{m}(-1)^{k+l} \delta_{k}^{m+1} \circ h_{l}+\sum_{k=l+2}^{m+1} \sum_{l=0}^{m-1}(-1)^{k+l} \delta_{k}^{m+1} \circ h_{l-1}+ \\
& +\sum_{k=0}^{m} \sum_{l=0}^{m-1}(-1)^{k+l} h_{l}^{m} \circ \delta_{k}^{m}=\mathrm{Id}-\mathrm{Inc}^{m} \circ \mathrm{Str}^{m}+ \\
& +\sum_{k=0}^{l-1} \sum_{l=1}^{m}(-1)^{k+l} h_{l-1} \circ \delta_{k}^{m}+\sum_{k=l+2}^{m+1} \sum_{l=0}^{m-1}(-1)^{k+l} h_{l} \circ \delta_{k-1}^{m}+ \\
& +\sum_{k=0}^{m} \sum_{l=0}^{m-1}(-1)^{k+l} h_{l}^{m} \circ \delta_{k}^{m}=\mathrm{Id}-\operatorname{Inc}^{m} \circ \mathrm{Str}^{m} \\
& -\sum_{k=0}^{p} \sum_{p=0}^{m-1}(-1)^{k+p} h_{p} \circ \delta_{k}^{m}-\sum_{k=p+1}^{m} \sum_{p=0}^{m-1}(-1)^{k+p} h_{p} \circ \delta_{k}^{m}+ \\
& +\sum_{k=0}^{m} \sum_{l=0}^{m-1}(-1)^{k+l} h_{l}^{m} \circ \delta_{k}^{m}=\mathrm{Id}-\mathrm{Inc}^{m} \circ \mathrm{Str}^{m}- \\
& -\sum_{k=0}^{m} \sum_{p=0}^{m-1}(-1)^{k+p} h_{p}^{m} \circ \delta_{k}^{m}+\sum_{k=0}^{m} \sum_{l=0}^{m-1}(-1)^{k+l} h_{l}^{m} \circ \delta_{k}^{m}= \\
& =\mathrm{Id}-\mathrm{Inc}^{m} \circ \mathrm{Str}^{m} \text {, }
\end{aligned}
$$

where we used the relations (18) - (22). Thus, we have a quasi-isomorphisms between the two chain complexes, which means that the two Hochschild homologies are isomorphic.

\section{Final REMARKS}

The basic ideas of these proof are "super"-versions of the classical, ungraded proof (see 8]). They amount to an unpublished result of R.K. Dennis (whence the name). We notice, however, to avoid confusions, that the term "Dennis trace" is also used for another map (related to the generalized trace, also introduced by Dennis), establishing a connection between algebraic K-theory and Hochschild homology (see [8]).

The Dennis supertraces can be used, as well, to provide a proof of the Morita invariance of the cyclic homology of the superalgebras (see [2]). We also managed to prove, recently, the general Morita invariance of Hochschild homology of superalgebras, not only for the case of supermatrices (see [1]). We used there a spectral sequence argument. Probably the 
more "economical" tools used by McCarthy ([7], see also the book of Loday [5]) can be adapted, as well, to the super-case.

\section{ACKNowledgments}

This paper was started when the author was a postdoc at the Universitá Politecnica delle Marche from Ancona, in the framework of the European Research Training Network "Geometric Analysis". The author would like to acknowledge both the financial support of the European Commission and the human and scientific support of Professor Nicolae Teleman.

\section{REFERENCES}

[1] Blaga, P.A., On the Morita invariance of the Hochschild homology for superalgebras, Studia Univ. "Babeş-Bolyai", (Mathematica), vol. LI (2006), no. 1, 41-48

[2] Blaga, P.A., On the Cyclic Homology of Supermatrices, in Proceedings of the Algebra Symposium, edited by S. Breaz and C. Săcărea, Editura EFES, Cluj-Napoca, 2006, 9-16

[3] Bartocci, C., Bruzzo, U., Hernandez-Ruiperez, D., The Geometry of Supermanifolds, Kluwer, 1992

[4] Kassel, C., A Künneth formula for the cyclic homology of $\mathbb{Z} / 2$-graded algebras, Math. Ann., 257 (1986), 683-699

[5] Loday, J.L., Cyclic Homology, Springer, 1992

[6] Manin, Yu., Complex Geometry and Gauge Theory, Springer Verlag, 1988

[7] McCarthy, R. Morita equivalence and cyclic homology, C.R. Acad. Sci. Paris Ser. I Math., volume 307 (1988), no. 6, 211-215

[8] Rosenberg, J., Algebraic K-Theory and Its Applications, Springer Verlag, 1994

University of Cluj-Napoca, Faculty of Mathematics and Computer Sciences, 1, KogăLniceanu Street, 400084 Cluj-Napoca, Romania

E-mail address: pablaga@cs.ubbcluj.ro 